EVOLUTION DE WUCHERERIA BANCROFTI ET W. MALAYI CHEZ AËDES (STEGOMYIA) AEGYPTI

ET A. (S.) ALBOPICTUS

Par H. GALLIARD

Le rôle d'Aëdes (Stegomyia) xgypti dans la transmission de la filaire de Bancroft a fait l'objet de recherches, avec des résultats divers, dans toutes les régions tropicales du globe. C'est ce qui nous a incité à étudier le rôle de ce culicidé. Nous avons publié les premiers résultats obtenus dès 1938 au Tonkin (C.R. Soc. Biol., CXXVIII, p. 1111), dans le delta du Fleuve Rouge, où il est extrêmement commun. Aëdes (Stegomyia) albopictus a une distribution géographique moins vaste, mais, localement, nous le considérons comme plus commun et encore mieux adapté à la vie domestique que $A$. ærgypti.

Par ailleurs, nous avons recherché si ces culicidés pouvaient permettre l'évolution de Filaria malayi, dont nous avons démontré en 1936 l’existence et la fréquence dans ce pays.

\title{
Wuchereria bancrofti
}

Après un certain nombre d'essais infructueux, au cours desquels les microfilaires dégénérèrent rapidement et disparurent vers le $4^{\circ}$ ou le $6^{\circ}$ jour, nous avons pu obtenir des formes évolutives chez les deux espèces d'Aëdes.

Dans le cas 239, le malade, hospitalisé pour orchite et hydrocèle chyleuse à l'hôpital de Nam Dinh, présentait 30 microfilaires par $10 \mathrm{~mm}^{3}$ de sang à $11 \mathrm{~h}$. du soir. L'évolution débute de même chez les deux Aëdes, comme chez Anopheles hyrcanus, mais s'arrête le $6^{\circ}$ jour chez $A$. albopictus et se poursuit chez $A$. ægypti jusqu'au $9^{\circ}$ jour : 1 exemplaire sur 5 est trouvé infesté avec 5 larves dans la tête et 2 dans la trompe. Il est à noter que, le $5^{\circ}$ jour, chez 4 sur 4 A. albopictus et 3 sur $5 \mathrm{~A}$. xgypti, on a trouvé des formes évolutives, 4 sur $8 \mathrm{~A}$. hyrcanus utilisés se sont infestés, dont un jusqu'au stade ultime.

Ann. de Parasitologie, T. XXII, n ${ }^{\circ 5} 1-2,1947$, p. 30-35. 
Dans le cas 331 , lie malade provenait des environs de Hanoï (prov. de Hadong) et se trouvait à l'hôpital Yersin pour hématochylurie. Son sang présentait 80 microfilaires par $10 \mathrm{~mm}^{3}$ à minuit. Les formes évolutives apparurent assez nombreuses chez les deux espèces les $5^{\circ}$ et $6^{\circ}$ jours, mais, à partir de ce moment, elles disparurent complètement. $4 \mathrm{~A}$. ægypti sur $40,3 \mathrm{~A}$. albopictus sur 50 présentèrent ces formes, alors que 2 A. hyrcanus sur 6 s'infestèrent.

Dans le cas 990 (hôpital R.-Robin à Bach, mai), le malade, hématochylurique, avait 20 microfilaires par $10 \mathrm{~mm}^{3}$ de sang à minuit. 27 exemplaires d'A. xgypti, 19 d'A. albopictus et 10 de Culex fatigans furent gorgés sur lui. A. albopictus ne s'infesta pas. Chez $A$. ægypti, rien ne fut trouvé jusqu'au $16^{\circ}$ jour, mais le $17^{\circ}$ jour, sur 7 spécimens, 2 furent trouvés infestés : l'un avec une larve sortant de la trompe, l'autre avec 14 larves dans le thorax, la tête et la trompe. Sur les 10 Culex, 2 sur 2 présentaient le $8^{\circ}$ jour des formes en saucisse et, le $12^{\circ}$ jour, 6 sur 6 étaient porteurs de larves infestantes (25 larves chez l'un d'eux).

Enfin, dans l'expérience 1.002, le malade atteint de funiculite, à l'hôpital Yersin, présentait 11 microlifaires par $10 \mathrm{~mm}^{3}$ de sang à minuit. L'expérience fut faite avec $36 \mathrm{~A}$. xgypti et $34 \mathrm{~A}$. albopictus et dura jusqu'au $24^{\circ}$ jour. A aucun moment, ne furent trouvées de formes évolutives, sauf quelques microfilaires dégénérées dans le thorax.

$$
* *
$$

Si l'on se reporte aux nombreux essais qui ont été faits dans les différentes parties du monde, on constate que les échecs ont été nombreux.

Fulleborn (1908), Ashburn et Craig (1907), Francis (1912) à Charleston, Sweet (1924) à Brisbane, Walker (1924) en Australie, Heydon (1931) au Queensland, Lester (1932) au Nigeria n'ont obtenu aucun résultat. En 1922, F. W. Edwards, dans une revue critique, concluait que chez $A$. ægypti, le développement n'avait pas lieu.

Quelques auteurs ont constaté un développement partiel : Low (1901) à Ste-Lucie, Vincent à la Trinité (1901) (pas de développement au delà du 6 e jour), Lebredo (1905) et Bahr (1912) aux Fidji, Vevers (1924) en Guyane anglaise (microfilaires sans gaine dans le thorax), Mac Kenzie (1925) dans l'archipel de Cook (larves toutes mortes le $11^{\circ}$ jour), Barbeau (1928) à Maurice, Connal (1930) (33 pour 100 infectés, mais développement arrêté le $3^{\bullet}$ jour), Sundar Rao et Iyengar (1932) (quelques spécimens aux stades de début). Davis 
(1935) au Brésil, O'Connor et Hulse (1935) à Porto-Rico (toujours des échecs, dans quelques cas les embryons gagnent le thorax, mais dégénèrent de deuxième ou troisième jour), O'Connor et Beatty (1938) à Sainte-Croix.

Il en est de même pour Aëdes albopictus : James au Travancore (1900), Yamada (1927), Barbeau (1928), Hu (1935) en Chine (larves. mortes, chez 42 exemplaires), Brug (1939) à Kabaena (Célèbes) n'ont pas vu se produire l'évolution complète.

Seul, Hicks en Sierra Leone (1932) a obtenu l'évolution complète chez $A$. ægypti ; chez 39 spécimens vivants, le $14^{\circ}$ jour, il a vu une fois l'infestation de la trompe. Au cours de nombreuses expériences antérieures, il n'avait rien obtenu. Il considère le fait comme exceptionnel et n'a jamais vu d'infection natureile.

Par contre, Flu (1928) à Paramaribo trouve une infection naturelle chez 8,3 pour 100 des spécimens capturés, et, seul de son avis, admet que cette espèce s'infeste aussi facilement que Culex fatigans.

Des recherches récentes de Byrd, St-Amant et Bromberg (1945) aux Samoa confirment la non-réceptivité d'A. xgypti. Mais des résultats positifs ont été obtenus en Amérique par Newton, Wright et Pratt en 1945. Ces auteurs, à Porto-Rico, ont obtenu des larves infestantes chez 5 pour 100 des $A$. ægypti (126 spécimens utilisés, 102 disséqués après le $9^{\circ}$ jour, 8 larves infestantes chez 4 , une forme prémature chez un autre).

Plus récemment encore (1946), Henrard, Peel, Wanson au Congo belge ont pu obtenir, sur einq Aëdes ægypti disséqués le $10^{\circ}$ jour, deux fois des larves infestantes (14 et 10 larves respectivement, dans le thorax). Dans un second essai, avec 20 spécimens, 5 disséqués le $17^{\circ}$ jour hébergeaient des formes infestantes dans la trompe. dans la tête, le thorax et l'abdomen.

\section{Wuchereria malayi}

En 1938, nous avions essayé d'infester, mais sans succès, les deux Aëdes sur des porteurs de microfilaria malayi. Nous avons répété ces essais en 1942 et réussi à infester les deux espèces.

Le malade hospitalisé pour une affection d'ordre chirurgical ne présentait pas de signes cliniques de filariose. Il avait 11 microfilaires par $10 \mathrm{~mm}^{3}$ de sang périphérique à 23 heures. 22 Aëdes xgypti et $40 \mathrm{~A}$. albopictus ont été gorgés sur lui à cette heure.

Chez un spécimen d'A. ægypti, il a été trouvé le $9^{\circ}$ jour une forme prémature et une forme infestante dans la tête. Le même jour, chez A. albopictus, il y avait deux formes prématures et deux infestantes, 
l'une dans le thorax, l'autre déjà engagée dans la trompe. Le $10^{\circ}$ jour, chez A. albopictus, il y avait une larve mûre, mais morte, dans le labium.

C'est à notre connaissance la première fois qu'on réussit l'infestation de ces deux espèces avec $F$. malayi et c'est la première fois que l'on obtient l'évolution complète d'une filaire humaine chez A. albopictus.

L. C. Feng à Huchow (1934) a trouvé chez 3 A. Albopictus sur 28, avant le $4^{\circ}$ jour, des microfilaires normales et actives, soit dans l'estomac, soit dans les muscles thoraciques. Après le $4^{\circ}$ jour, le résultat des dissections a été toujours négatif. S. M. K. Hu (1942) à Shangaï a répété ces essais avec A. albopictus. Sur 180 spécimens gorgés sur des malades peu parasités, 2 seulement contenaient une seule larve immature, morte et encapsulée. Dans un cas d'infestation forte, 98 Aëdes sur 178 hébergeaient dans l'abdomen des larves au premier stade, mortes et encapsulées.

Discussion. - Les résultats que nous avons obtenus montrent que l'on peut obtenir l'évolution complète jusqu'au stade infestant de $W$. bancrofti chez $A$. xgypti, et de $W$. malayi chez $A$. xgypti et A. albopictus.

Lorsque l'expérience réussit, la proportion des $A$. æxgypti qui s'infeste est relativement importante : 1 sur 15 dans le cas 239,2 sur 27 dans le cas 990 , ce qui ferait 6,66 pour 100 pour le premier et 7,4 pour le second. La proportion serait encore plus importante si l'on tenait compte seulement du nombre des moustiques encore vivants, le $9^{\circ}$ jour. Ces chiffres sont incomparablement moins élevés que ceux obtenus avec Anopheles hyrcanus et surtout Culex fatigans que nous avons dans chaque cas infestés avec les Aëdes : sur 10 C. fatigans (cas 990 ), 6 sur 6 disséqués le $12^{\circ}$ jour ont présenté des larves infestantes. Sur 5 A. hyrcanus (cas 239), une fois ces formes ont été trouvées.

Il est cependant vrai que dans certaines expériences, l'évolution complète ne s'obtient pas. Dans le cas 331 , l'évolution n'a pas dépassé le stade en saucisse chez $50 \mathrm{~A}$. albopictus et $40 \mathrm{~A}$. rgypti et a nettement avorté. Dans le cas 1.002 , chez $36 \mathrm{~A}$. xgypti et 34 . A. albopictus, l'échec a été complet, aucune forme évolutive n'ayant été trouvée.

On peut se demander à quoi tient la variabilité de ces résultats, étant donné, bien entendu, que l'on a expérimenté dans des conditions optima de température $\left(28-32^{\circ} \mathrm{C}\right)$ et d'humidité. Il semble tout d'abord que le nombre des moustiques utilisés n'entre pas en

Ann. de Parasitologie, t. XXII, nos $1-2 ; 1947$.

3. 
ligne de compte. Dans le cas de $W$. bancrofti, nous n'avons obtenu aucun résultat en expérimentant avec 90 spécimens une fois et 70 une autre fois. Par contre, dans deux autres cas, avec 30 et 10 spécimens respectivement, nous avons réussi. Nous avons dit plus haut que $\mathrm{Hu}$ n'avait pas obtenu de résultats en utilisant un nombre important de spécimens. Il est par ailleurs nécessaire (si on expérimente à un température de $28^{\circ}-32^{\circ}$ ) de sacrifier tous les moustiques au plus tard le $20^{\circ}$ jour. A partir de ce moment, en effet, les larves infestantes sont éliminées plus ou moins rapidement.

Par ailleurs, l'importance de l'infestation humaine peut entrer en ligne de compte et l'on sait que le pourcentage des moustiques infectés est proportionnel, dans une certaine mesure, au nombre des microfilaires existant dans le sang périphérique. Cependant, ce facteur n'a pas une influence déterminante, même chez les culicidés peu réceptifs comme Aëdes rgypti et Aëdes albopictus. Nous avons constaté, en effet, que dans le cas de $A$. æxypti, nos malades hébergeaient respectivement 80 (insuccès), 20 et 30 (succès) microfilaires de $W$. bancrofti pour $10 \mathrm{~mm}^{3}$ de sang. Dans le cas de $W$. malayi, il n'y avait que 11 microfilaires pour $10 \mathrm{~mm}^{3}$, alors que $\mathrm{Hu}$ 'a pas obtenu de résultats avec des malades fortement infestés.

En 1938, nous avions émis l'hypothèse qu'il s'agissait peut-être de conditions favorables tenant au malade porteur de microfilaires. Peut-être existe-t-il aussi des races locales de parasites s'adaptant plus ou moins facilement à tel ou tel vecteur? Des recherches récentes montrant qu'aux îles Samoa (Byrd et coll., 1945), A. ægypti est réfractaire, alors qu'à Porto-Rico (Newton, Wright et Pratt, 1945), chez 5 pour 100 des $A$. ægypti, gorgés expérimentalement, on observe un développement complet jusqu'au stade infestant, semblent plaider en faveur de cette opinion.

Ces considérations pourraient d'ailleurs s'étendre à d'autres vecteurs, comme Culex fatigans en particulier. Henrard, Peel et Wanson considèrent que, au Congo belge, l'évolution de W. bancrofti se fait beaucoup mieux chez Anopheles funestus et A. gambiæ que chez C. fatigans.

Il est naturel que ce culicidé soit moins réceptif que d'autres dans les îles du Pacifique, comme l'ont encore montré récemment Byrd, St-Amant et Bromberg, où l'existence d'une race biologique particulière ( $W$. bancrofti apériodique) est certaine. Par contre, nous avons constaté que $C$. fatigans est extrêmement sensible à l'infestation expérimentale, beaucoup plus qu'A. hyrcanus, et il est certainement le vecteur le plus efficace de la filariose dans le delta du Tonkin. 


\section{RÉSUMÉ}

L'évolution des larves de Wuchereria bancrofti peut s'effectuer dans certaines circonstances chez Aëdes ægypti jusqu'au stade infestant. Dans d'autres cas, ou l'évolution avorte ou bien les larves restent au stade de début et dégénèrent. Chez Aëdes albopictus, on ne trouve que les stades de début.

L'évolution de $W$. malayi a pu être obtenue chez $A$. ægypti et A. albopictus. Dans des conditions semblables de température, le nombre des microfilaires de sang périphérique intervient peu, et il semble que la divergence des résultats observés puisse être due soit à l'hôte, soit à la race des parasites infestants.

\section{BibLIOGRAPHIE (1)}

FLU (P.-C.). - Verslag van een studiureis naar Suriname Nederlandsch Guyana. Acta Leidensia Schola Med. Trop., III, 1928, p. 1.

Galliard (H.). - Evolution complète de Filaria bancrofti chez Aedes (Stegomyia) rgypti. C.R. Soc. Biol.; CXXVIII, 1938, p. 1111.

- Evolution complète de Filaria malayi chez Aedes agypti et A, albopictus. Rev. med. Fr. Extr. Orient, XIX, 1941, p. 420.

Henrard (C.), Perl (E.) et Wanson (M.). - Quelques localisations de Wuchereria bancrofti au Congo belge. Cycle de développement chez Culex fatigans, Anopheles funestus, Aedes agypti, Anopheles gambir. Rec. Trav. Sci. Med. Congo belge, 1946, $\mathrm{n}^{\circ}$ 5, p. 212.

Hicks (E.-P.). - The transmission of Wuchereria bancrofti in Sierra Leone. Ann. trop. Med. and parasit., XXVI, 1932, p. 407.

Newton (W.-L.), Wright (W.-H.) et Pratt (I,). - Experiences to determine potential mosquito vectors of Wuchereria bancrofti in continental United States. Amer. Jl. Trop. med., XXV, 1945, p. 253.

(1) Nous ne mentionnons dans cette liste que les auteurs qui ont obtenu le développement complet des larves de $W$. bancrofti.

\section{Laboratoire de parasitologie} Faculté de médecine de Hanoï 